\title{
Electronic transport in modulation-doped InSb quantum well heterostructures
}

\author{
J. M. S. Orr, ${ }^{1,2}$ A. M. Gilbertson, ${ }^{1,3}$ M. Fearn, ${ }^{1}$ O. W. Croad, ${ }^{1}$ C. J. Storey, ${ }^{1}$ L. Buckle, ${ }^{1}$ M. T. Emeny, ${ }^{1}$ P. D. Buckle,,${ }^{1, *}$ \\ and T. Ashley ${ }^{1}$ \\ ${ }^{1}$ QinetiQ, Malvern WR14 3PS, United Kingdom \\ ${ }^{2}$ School of Electrical and Electronic Engineering, University of Manchester, Manchester M60 1QD, United Kingdom \\ ${ }^{3}$ Department of Physics, Imperial College, London SW72AZ, United Kingdom
}

(Received 22 November 2007; revised manuscript received 6 February 2008; published 28 April 2008)

\begin{abstract}
The mobility and carrier concentration of a number of InSb-based modulation-doped quantum well heterostructures are examined over a range of temperatures between 4.5 and $300 \mathrm{~K}$. Wide well $(30 \mathrm{~nm})$ and narrow well $(15 \mathrm{~nm})$ structures are measured. The temperature dependent mobilities are considered within a scattering model that incorporates polar optical and acoustic phonon scatterings, interface roughness scattering, and scattering from charged impurities both in the three-dimensional background and within a distributed "quasitwo-dimensional" doping layer. Room temperature mobilities as high as $51000 \mathrm{~cm}^{2} / \mathrm{V}$ s are reported for heterostructures with a carrier concentration of $5.8 \times 10^{11} \mathrm{~cm}^{-2}$, while low-temperature mobility (below $40 \mathrm{~K}$ ) reaches $248000 \mathrm{~cm}^{2} / \mathrm{V}$ s for a carrier concentration of $3.9 \times 10^{11} \mathrm{~cm}^{-2}$. A Schrödinger-Poisson model is used to calculate band structures in the material and is shown to accurately predict carrier concentrations over the whole temperature range. Low-temperature mobility is shown to be dominated by remote ionized impurity scattering in wide well samples and by a combination of ionized impurity and interface roughness scattering in narrow well samples.
\end{abstract}

DOI: 10.1103/PhysRevB.77.165334

PACS number(s): 72.80.-r, 73.21.-b

\section{INTRODUCTION}

Electronic transport in remotely doped heterostructures is a subject of significant interest in the fields of high-speed electronics (high electron mobility transistors) ${ }^{1}$ and ballistic mesoscopic devices, ${ }^{2}$ as well as for magnetic sensor applications, ${ }^{3,4}$ in which a high electron saturation velocity leads to enhanced magnetic sensitivity. In devices for which ballistic transport is a requirement, it is necessary to optimize both the carrier mobility and the concentration in the twodimensional electron gas (2DEG) since the ballistic electron mean free path, $l_{e}$, at low bias, is directly proportional to the mobility, $\mu$, and the square root of the carrier concentration, $n_{e},{ }^{5}$

$$
l_{e}=\left(\frac{h}{e \sqrt{2 \pi}}\right) \mu \sqrt{n_{e}} .
$$

Given the large lattice mismatch between InSb and its potential alloy materials, high-quality growth is challenging and InSb-based heterostructures are, as a result, not yet widely studied. However, when compared to far more common GaAs-based materials systems, InSb-based heterostructures offer several intrinsic advantages, such as the lowest bulk electron effective mass of any III-V semiconductor $\left(0.014 m_{e}\right)$, a large dielectric constant $\left(16.8 \epsilon_{0}\right)$, the narrowest band gap of any III-V semiconductor $(170 \mathrm{meV}$ at room temperature), and an exceptionally large Landé $g$ factor $(-51)$, which could lead to important applications in spin-tocharge readout ${ }^{6}$ and selective qubit addressing ${ }^{7}$ in quantum computing. Electronic behavior in GaAs has been well understood for many years, but it is only comparatively recently that high-quality InSb heterostructures have been fabricated and characterized. ${ }^{8}$ The resulting electron mobilities are sufficient to exhibit ballistic transport effects in mesoscopic structures, such as one-dimensional (quantum wire) transport in etched ${ }^{9}$ and soft-confined (gated) ${ }^{10}$ quantum point contacts. In this paper, we report the growth and characterization of high-mobility $\mathrm{InSb} / \mathrm{Al}_{x} \mathrm{In}_{1-x} \mathrm{Sb}$ heterostructures with a single Te delta-doping plane, which have been shown to operate under Schottky-barrier surface gating ${ }^{1,11,12}$ for device applications. We include results of the effects induced by variation in spacer thickness between the quantum well and the single Te-doping plane, and we present results on the variation of the width of the quantum well and the composition of barrier materials and dopant concentration, with a view to optimizing the materials system for highspeed or mesoscopic transport. We use a transport lifetime model, which is based on the relaxation time approximation, to examine the contributions of individual scattering mechanisms on the electron transport.

\section{GROWTH AND FABRICATION}

The $\mathrm{InSb} / \mathrm{Al}_{x} \mathrm{In}_{1-x} \mathrm{Sb}$ heterostructure was grown by solidsource molecular beam epitaxy (MBE) on a semi-insulating GaAs substrate. Two types of structure were grown: one with a $30 \mathrm{~nm} \mathrm{InSb}$ quantum well layer [Fig. 1(a)] and the other with a $15 \mathrm{~nm}$ InSb quantum well layer [Fig. 1(b)]. The wider well samples consist of, in growth order, an accommodation layer, a $3 \mu \mathrm{m} \mathrm{Al}_{0.10} \mathrm{In}_{0.90} \mathrm{Sb}$ buffer layer, a $30 \mathrm{~nm} \mathrm{InSb}$ quantum well, and a $50 \mathrm{~nm}$ upper barrier layer of $\mathrm{Al}_{0.15} \mathrm{In}_{0.85} \mathrm{Sb}$. The narrower well samples consist of the same accommodation layer, a wider band gap $3 \mu \mathrm{m}$ buffer layer of $\mathrm{Al}_{0.15} \mathrm{In}_{0.85} \mathrm{Sb}$, and a $15 \mathrm{~nm}$ InSb quantum well followed by a $50 \mathrm{~nm} \mathrm{Al} \mathrm{Al}_{0.20} \mathrm{In}_{0.80} \mathrm{Sb}$ top barrier. Within the upper barrier layer, which is separated from the well by a spacer, $d$, is a Te $\delta$-doping plane. Postgrowth secondary ion mass spectroscopy (SIMS) studies show Te-dopant atoms distributed through the top barrier with an exponential decay in concentration in the growth direction, away from the quantum well 

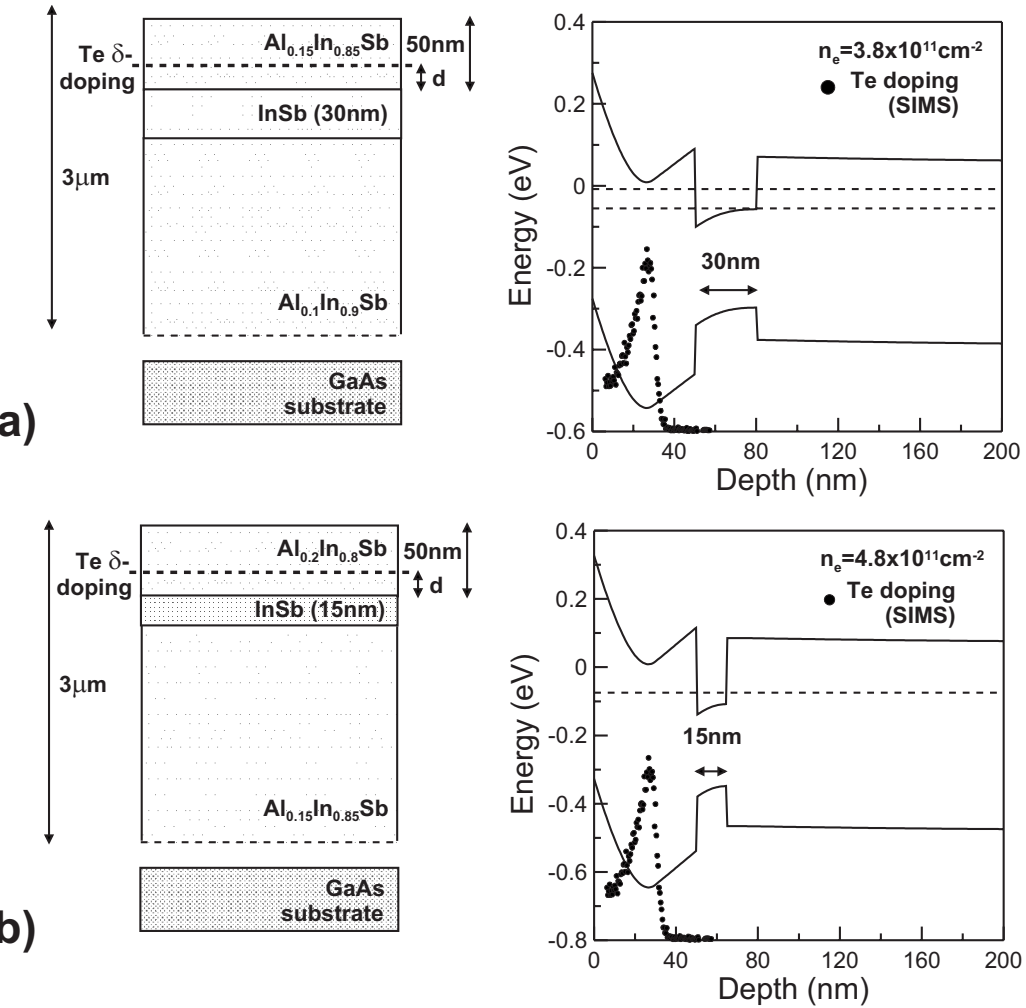

FIG. 1. Layer structures for (a) 30 and (b) 15 $\mathrm{nm}$ quantum well heterostructure samples indicating the barrier compositions and dimensions in the growth direction. Schrödinger-Poisson solutions for 30 and $15 \mathrm{~nm}$ layers at $10 \mathrm{~K}$ (right hand side) showing occupied confined states (dashed lines) below the Fermi energy $\left(E_{f}=0 \mathrm{meV}\right)$ and SIMS profile of Te distribution. (this is superimposed onto the Schrödinger-Poisson solutions in Fig. 1).

The material is intentionally depletion mode and is designed to operate under negative biases supplied by Schottky contacts on the surface. The doping and alignment are such that the equilibrium Fermi energy falls above the first confined state, so that the conduction band bound states are populated at all temperatures. These layers form a type-I heterostructure, which provides confinement for both electrons and holes in the channel layer. Schrödinger-Poisson solutions for the band structure of these layers are shown under zero surface bias in Fig. 1. Wide and narrow well structures are presented in Fig. 1(a), right hand side, for a carrier concentration of $3.8 \times 10^{11} \mathrm{~cm}^{-2}$ and in Fig. 1(b), right hand side, for a carrier concentration of $4.8 \times 10^{11} \mathrm{~cm}^{-2}$. Midgap pinning is assumed at the surface, ${ }^{11,12}$ and the spatially varying Te concentration is taken into account by calculating the Schrödinger-Poisson solutions with a Te distribution fitted to the SIMS profiles shown in Fig. 1.

Ti/Au e-beam evaporated Ohmic contacts were defined by optical lithography and a lift-off process. The devices were isolated by wet-chemical etching down to the substrate to eliminate pad-to-pad conduction in the barrier layers. Shallow contact technology is employed to ensure that transport is via the $2 \mathrm{D}$ channel only. Evidence for single subband solely 2D transport is clearly seen in low-temperature Shubnikov-de Haas measurements (not shown) consistent with a large subband spacing $\left(>k_{B} T\right)$ as a consequence of strong confinement and light effective mass.

\section{MOBILITY AND CARRIER CONCENTRATION IN $\mathrm{InSb} / \mathrm{Al}_{x} \mathrm{In}_{1-x} \mathrm{Sb}$ HETEROSTRUCTURES}

Hall measurements were carried out on a variety of heterostructure samples over a range of temperatures between 4.5 and $300 \mathrm{~K}$ by using a liquid helium bath cryostat. The samples were mounted on ceramic leadless chip carriers and connected via triax shielding to a HP4156B semiconductor parameter analyzer. The sample space sits between the poles of a $450 \mathrm{mT}$ electromagnet. Hall bar samples with a length:width aspect ratio of 5:1 were assessed for longitudinal resistivity by sweeping the longitudinal current between -10 and $+10 \mu \mathrm{A}$ in zero field and recording the voltage drop in a four-contact measurement, eliminating lead resistances. The Hall resistance was then determined by using a lateral voltage measurement in the presence of a swept magnetic field between 100 and $300 \mathrm{mT}$ under a current of $10 \mu \mathrm{A}$ to limit sample heating. Growth details of the samples presented in depth in this study, for which mobility and carrier concentration measurements were taken as a function of temperature, are presented in Table I. The wide well samples are denoted "W" and the narrow well samples are denoted "N."

Figure 2 shows the carrier concentration taken from Hall measurements of sample $\mathrm{W} 2$ as a function of temperature. At $4.5 \mathrm{~K}$, the carrier density in the 2DEG is $3.8 \times 10^{11} \mathrm{~cm}^{-2}$ and rises to around $5.4 \times 10^{11} \mathrm{~cm}^{-2}$ at $300 \mathrm{~K}$. The lowtemperature carrier concentration is used to generate an accurate Schrödinger-Poisson model for the structure that allows the temperature dependence of the carrier concentration to be modeled. The modeled points in Fig. 2 (filled circles) demonstrate a good fit with the data, which indicates that the Schrödinger-Poisson model provides a realistic representa- 
TABLE I. InSb/ $\mathrm{Al}_{x} \mathrm{In}_{1-x} \mathrm{Sb}$ heterostructure samples presented in this paper.

\begin{tabular}{lcc}
\hline \hline Sample & $\begin{array}{c}\text { Well width } \\
(\mathrm{nm})\end{array}$ & $\begin{array}{c}\text { Spacer } \\
(\mathrm{nm})\end{array}$ \\
\hline W1 & 30 & 25 \\
W2 & 30 & 20 \\
N1 & 15 & 20 \\
N2 & 15 & 10 \\
N3 & 15 & 5 \\
\hline \hline
\end{tabular}

tion of the electronic band structure and carrier densities over the studied temperature range. The origin of the changing carrier concentration with temperature derives from a combination of the temperature dependence of the band structure and the thermal activation of higher energy subbands. Although we cannot completely exclude the possibility of unionized donors, the good agreement with experimental data shown in Fig. 2, assuming fully ionized donors, gives a high level of confidence: the degree of donor ionization is not a major contribution to the channel carrier density. For the samples reported here, the measured carrier concentrations vary between $(3.7-5.1) \times 10^{11} \mathrm{~cm}^{-2}$ at $10 \mathrm{~K}$ and $(5.1-8.0) \times 10^{11} \mathrm{~cm}^{-2}$ at $300 \mathrm{~K}$ for the wide well samples and between $(2.8-5.7) \times 10^{11} \mathrm{~cm}^{-2}$ at $10 \mathrm{~K}$ and $(3.7-6.0)$ $\times 10^{11} \mathrm{~cm}^{-2}$ at $300 \mathrm{~K}$ for the narrow well samples.

Figure 3 shows the Hall mobility for a range of narrow well heterostructures as a function of temperature, with 20 , 10 , and $5 \mathrm{~nm}$ spacers between the quantum well and the doping plane. Below $50 \mathrm{~K}$, the mobility of all samples remains roughly constant. The low-temperature mobilities are 111000,72300 , and $63600 \mathrm{~cm}^{2} / \mathrm{V}$ s for the 20, 10, and 5 $\mathrm{nm}$ spacer samples, respectively. Above $50 \mathrm{~K}$, the mobility drops off with temperature, with the curves converging above $250 \mathrm{~K}$. At $300 \mathrm{~K}$, the mobilities of all of the narrow well samples are approximately $40000 \mathrm{~cm}^{2} / \mathrm{V} \mathrm{s}$. The inset in Fig. 3 shows the low-temperature mobilities as a function

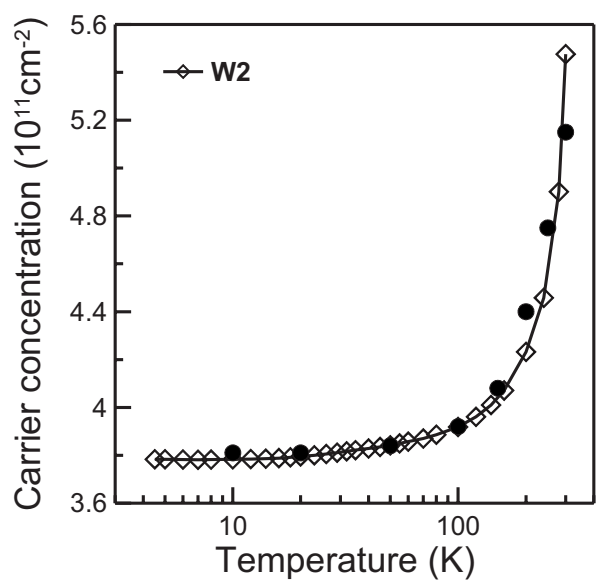

FIG. 2. Measured carrier concentration of W2 as a function of temperature. The carrier concentrations calculated from Schrödinger-Poisson solutions for this structure are shown as filled circles.

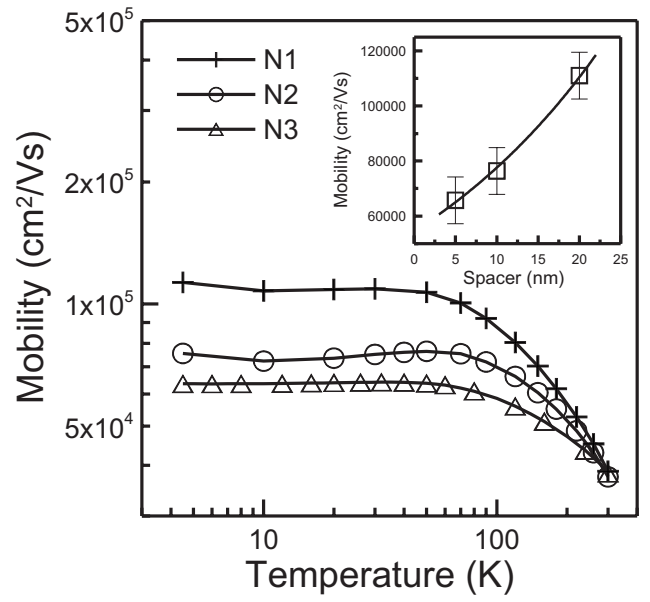

FIG. 3. Temperature-dependent mobility of narrow $(15 \mathrm{~nm})$ well InSb heterostructures with 20, 10, and $5 \mathrm{~nm}$ spacers from Hall measurements taken between 4.5 and $300 \mathrm{~K}$. The inset shows lowtemperature mobility as a function of spacer separation (open squares). These data are consistent with exponential behavior (solid line).

of spacer separation. The error bars are consistent with the spread of data for similar devices of the same wafer growth, from which an exponential fit between mobility and spacer is demonstrated (solid line).

The temperature dependence of the mobility in wide well samples is shown in Fig. 4. The two samples presented show low-temperature mobilities far in excess of those measured for the narrower wells, with $10 \mathrm{~K}$ mobilities of $248000 \mathrm{~cm}^{2} / \mathrm{V} \mathrm{s}$ for the $25 \mathrm{~nm}$ spacer sample (W1) and $214600 \mathrm{~cm}^{2} / \mathrm{V} \mathrm{s}$ for the $20 \mathrm{~nm}$ spacer sample (W2). The mobility in both samples remains constant below $40 \mathrm{~K}$ (a slightly lower temperature than that observed in the narrower well samples); again, the mobilities converge at higher temperatures, with $300 \mathrm{~K}$ mobilities of $52000 \mathrm{~cm}^{2} / \mathrm{V} \mathrm{s}$ (W1) and $48000 \mathrm{~cm}^{2} / \mathrm{V} \mathrm{s}$ (W2).

The $300 \mathrm{~K}$ mobilities and carrier concentrations of the samples named in Table I, together with data from a number of additional layers of similar construction, are shown in Fig.

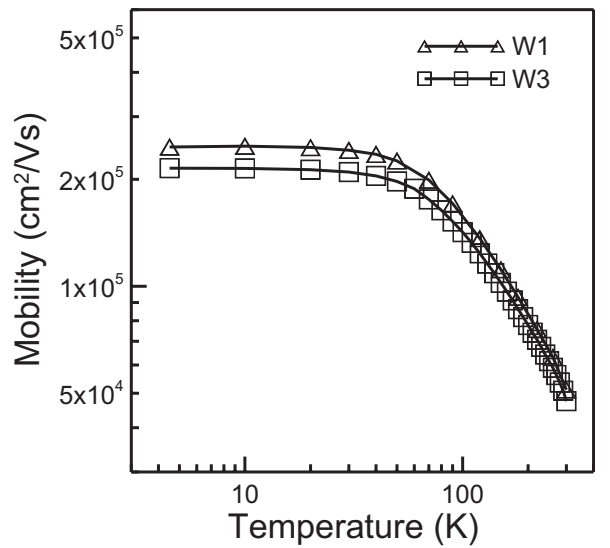

FIG. 4. Temperature-dependent mobility of wide $(30 \mathrm{~nm})$ well InSb heterostructures with 25 and $20 \mathrm{~nm}$ spacers from Hall measurements taken between 4.5 and $300 \mathrm{~K}$. 


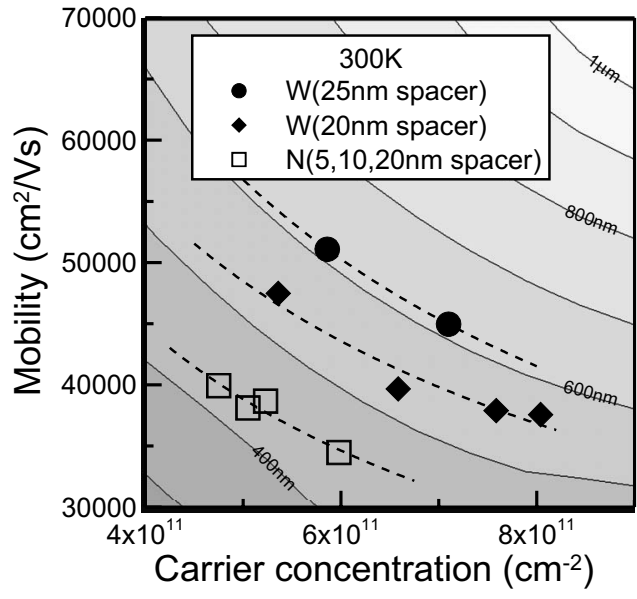

FIG. 5. Mobility versus carrier concentration for a range of heterostructures at room temperature showing a general correlation between mobility and carrier concentration for particular layer designs. A general trend of increased mobility for lower carrier concentration is observed and indicated by a dashed line. The data are overlayed onto a contour map of ballistic transport lengths from Eq. (1).

5, displaying a general trend in which increased mobilities are achievable for a lower carrier concentration. These trends are indicated by dashed lines, and it is observed that they roughly follow the ballistic length contours. The highest recorded mobility at $300 \mathrm{~K}\left(51100 \mathrm{~cm}^{2} / \mathrm{V} \mathrm{s}\right)$ is found for a wide well sample (W1) with a large $(25 \mathrm{~nm})$ spacer. The data are overlayed onto a contour map of ballistic transport lengths by following Eq. (1); this estimates the ballistic transport lengths for electrons in these heterostructures to be between 0.45 and $0.65 \mu \mathrm{m}$ at $300 \mathrm{~K}$.

The data in Fig. 5 show that the mobility in the wide well structures ranges from 37600 to $51100 \mathrm{~cm}^{2} / \mathrm{V} \mathrm{s}$, while in the narrow well structures the mobility varies between 34500 and $39900 \mathrm{~cm}^{2} / \mathrm{V} \mathrm{s}$ at $300 \mathrm{~K}$. It is also notable that the spacer distance in the narrow well layers seems to be of less significance to the room temperature transport characteristics of the samples.

\section{DISCUSSION AND TRANSPORT LIFETIME MODEL}

Within the transport formalism of the relaxation time approximation, the electron drift under applied fields is opposed by a scattering term, which is characterized by the momentum relaxation time, or transport lifetime, $\tau_{\mathrm{tr}}$. We recognize the limitation of this approximation, especially in the context of narrow-gap semiconductors; however, it does enable ready comparison of the relative importance of the various scattering mechanisms. A detailed theoretical treatment of scattering in this material is currently the subject of additional investigation. ${ }^{13}$ The scattering of an electron wave packet in a crystalline material consists of a variety of mechanisms that are individually well characterized and have temperature dependences that explain the overall characteristics observed in the mobility of particular heterostructures. It is important that at certain temperatures, such as the cryogenic temperature ranges, the mobility may be strongly dominated by one particular scattering mechanism. This can be used to engineer and, in general, maximize the mobility so as to increase the ballistic transport length or device performance.

The most significant scattering mechanisms are well recognized in III-V semiconductors, ${ }^{14}$ and the total transport lifetime, $\tau_{\text {tr }}$, can be calculated from the sum of individual scattering rates. These may be separated into two broad categories: phonon scattering - wherein perturbations to the lattice potential affect the electronic transport-and ionized impurity scattering-wherein electrons are influenced by the potentials of both remote and background impurities via the Coulomb interaction. This list is by no means exhaustive; indeed, scattering by uncharged material defects in the lattice and interface roughness scattering can also have a significant limiting effect on the mobility.

The principal contributions to phonon scattering are from polar phonons in the optical branch $\left(\tau_{\mathrm{po}}\right)$ and acoustic phonons $\left(\tau_{\mathrm{ac}}\right)$, while for impurity scattering, background impurities $\left(\tau_{\text {bgi }}\right)$ and remote ionized impurities in the doping layers $\left(\tau_{\text {rii }}\right)$ are important. The scattering rates from these processes may be derived from Fermi's golden rule.

Phonon scattering in semiconductors is well documented. ${ }^{15-21}$ The scattering of electrons by absorption of polar optical phonons in quantized 2D heterostructures has been considered by Price ${ }^{21}$ and Ridley. ${ }^{17}$ The characteristic lifetime for a quantum well of width $w$ is given by

$$
\frac{1}{\tau_{\mathrm{po}}}=\frac{e^{2} \omega_{0} N\left(\omega_{0}\right) m^{* 2} w}{4 \pi \epsilon_{p} \hbar^{2}}
$$

where $\epsilon_{p}^{-1}=\epsilon_{\infty}^{-1}-\epsilon_{s}^{-1}$ and $\epsilon_{\infty}$ and $\epsilon_{s}$ are the high-frequency and static dielectric constants, respectively. $\hbar \omega_{0}$ is the optical phonon energy and $N\left(\omega_{0}\right)$ gives the density of phonons, where

$$
N\left(\omega_{0}\right)=\left[\exp \left(\frac{\hbar \omega_{0}}{k_{B} T}\right)-1\right]^{-1} .
$$

In the above expression, it is assumed that there is no intersubband scattering; this is reasonable in systems wherein the subband spacing $\Delta E$ is sufficiently large that phonon absorption cannot induce a change of transverse mode.

The acoustic phonon scattering depends on the deformation potential $\Xi$ of the phonon in the lattice, the crystal density $\rho_{d}$, and the longitudinal sound velocity $v_{s} \cdot{ }^{14,16}$ Again, assuming that there is no intersubband scattering,

$$
\frac{1}{\tau_{\mathrm{ac}}}=\frac{3 m^{*} \Xi^{2} k_{B} T}{2 \hbar^{3} \rho_{d} v_{s}^{2} w} .
$$

Impurity scattering can be an important mechanism, particularly at low temperatures, at which phonon scattering is weak. Much work exists on the modeling of scattering from charged impurities, both localized and distributed throughout the lattice, within more common heterostructures, such as those based on GaAs. The analysis here follows that of Davies. ${ }^{22}$

The long-range Coulomb interaction between the ions and the electrons is described by the perturbation Hamiltonian $\hat{V}$. 
Following the Born approximation, the peturbation matrix element is expressed in terms of the two-dimensional Fourier transform of the scattering potential, $\widetilde{V}(q)$. From Fermi's golden rule, the scattering rate from $\mathbf{k}$ to $\mathbf{k}+\mathbf{q}$ is

$$
W_{k+q, k}=\frac{2 \pi}{A^{2} \hbar}|\tilde{V}(\mathbf{q})|^{2} \delta[\epsilon(\mathbf{k}+\mathbf{q})-\epsilon(\mathbf{k})],
$$

where the delta function is consistent with elastic scattering. The scattering angle is related to $|\mathbf{q}|=q$ via $\theta$ $=2 \sin ^{-1}(q / 2 k)$. The dependence of Eq. (5) on $1 / A^{2}$ reflects the fact that as the system grows, the scattering from a single impurity becomes less important. This factor disappears if we consider a two-dimensional density of scattering states and an average areal distribution of impurities; thus, the average scattering rate $\left\langle\tau_{\mathrm{tr}}\right\rangle$ is independent of device dimensions. Circularly symmetric scattering potentials introduce a transport lifetime, $\tau_{i}{ }^{22}$ where

$$
\frac{1}{\tau_{i}}=n_{\mathrm{imp}}^{2 \mathrm{D}} \frac{m^{*}}{2 \pi \hbar^{3} k_{f}^{3}} \int_{0}^{2 k_{f}}|\widetilde{V}(q)|^{2} \frac{q^{2} d q}{1-\sqrt{\left(q / 2 k_{f}\right)^{2}}},
$$

and since all conduction takes place close to the Fermi energy in a degenerate two-dimensional semiconductor, we can take $k=k_{f}=\sqrt{2 \pi n_{e}}$, where $n_{e}$ is the sheet carrier electron concentration. The integral in Eq. (6) runs between 0 and $2 k_{f}$, where a scattering wave vector of $q=2 k_{f}$ implies total backscattering of the electron. It then remains to substitute the appropriate expressions for the Fourier transformed scattering potentials for either remote ionized or background impurities.

First, the scattering from remote ionized impurities is considered for a two-dimensional sheet of charged impurities, $n_{\mathrm{imp}}^{2 \mathrm{D}}$, separated from the quantum well by a spacer $d$ by taking the Fourier transform of the Coulomb potential. The effects of screening are included by using the Thomas-Fermi approximation. The remote ionized impurity scattering rate for a two-dimensional sheet of impurity atoms at $d$ is then

$$
\frac{1}{\tau_{\mathrm{rii}}}=n_{\mathrm{imp}}^{2 \mathrm{D}} \frac{m^{*}}{2 \pi \hbar^{3} k_{f}^{3}}\left(\frac{e^{2}}{2 \epsilon_{0} \epsilon_{s}}\right)^{2} \int_{0}^{2 k_{f}} \frac{\exp (-2 q|d|)}{\left(q+q_{\mathrm{TF}}\right)^{2}} \frac{q^{2} d q}{\sqrt{1-\left(q / 2 k_{f}\right)^{2}}} .
$$

The transport lifetime associated with background impurities is found by integrating over a three-dimensional (3D) distribution of scattering centers as follows:

$$
\frac{1}{\tau_{\mathrm{bgi}}}=n_{\mathrm{imp}}^{3 \mathrm{D}} \frac{m^{*}}{2 \pi \hbar^{3} k_{f}^{3}}\left(\frac{e^{2}}{2 \epsilon_{0} \epsilon_{s}}\right)^{2} \int_{0}^{2 k_{f}} \frac{1}{\left(q+q_{\mathrm{TF}}\right)^{2}} \frac{q d q}{\sqrt{1-\left(q / 2 k_{f}\right)^{2}}} .
$$

The bounds of the integrals in Eqs. (7) and (8) refer to scattering angles between 0 and $180^{\circ}$. The assumption of a perfect delta-doping plane at $d$ leads to an overestimation of the scattering because, in reality, some surface transport of the Te atoms occurs during the growth. SIMS reveals a Te composition, which exponentially decays in concentration from $d$ toward the surface in the growth direction (see Fig. 1). To incorporate this into the ionized impurity model [Eq. (6)], the impurity distribution is considered a function of $d$, over the range $d_{s}$ (at the spacer) to $d_{0}$ (at the surface), so that we obtain a modified expression for ionized impurity scattering as follows:

$$
\begin{aligned}
\frac{1}{\tau_{\text {rii }}}= & \frac{m^{*}}{2 \pi \hbar^{3} k_{f}^{3}}\left(\frac{e^{2}}{2 \epsilon_{0} \epsilon_{s}}\right)^{2} \int_{d_{s}}^{d_{0}} \int_{0}^{2 k_{f}} n_{\mathrm{imp}}^{2 \mathrm{D}}(d) \\
& \times \frac{\exp (-2 q|d|)}{\left(q+q_{\mathrm{TF}}\right)^{2}} \frac{q^{2} d q d d}{\sqrt{1-\left(q / 2 k_{f}\right)^{2}}} .
\end{aligned}
$$

A further source of scattering, which is exclusive to heterostructure systems, comes from variation in the interfaces at the edges of the quantum well. Roughness at these interfaces causes the energy of the confined states to change as the width of the confinement changes. This has been studied by Hong et al. ${ }^{23}$ for a quasitriangular single heterointerface, where a strong dependence on well width is observed. For type-I aligned heterostructures, the dependence of the 2DEG ground-state energy on quantum well dimensions can be considered, to a first approximation, by referring to solutions to the time-independent Schrödinger equation in an infinite square well. ${ }^{24,25}$ The deviation from a perfectly flat interface is described by the function $\Delta(\mathbf{r})$, where $\mathbf{r}$ covers the $(x, y)$ plane in which the interface is defined. $\Delta$ is the rms average variation in the interface in the $z$ direction, which is usually of the order of a few monolayers for MBE-grown heterostructures, ${ }^{24}$ and $L$ is the characteristic extent of the roughness features. These quantities may be found by standard imaging techniques, such as cross-sectional transmission electron microscopy or atomic force microscopy.

The scattering rate is calculated from the Born approximation [as Eq. (5)] by using the square of the interface scattering matrix element given in Refs. 24-26,

$$
|\tilde{V}(\mathbf{q})|^{2}=\frac{\pi^{5} \hbar^{4} \Delta^{2} L^{2}}{m^{* 2} w^{6}} \exp \left(-\frac{q^{2} L^{2}}{4}\right) .
$$

As before [Eq. (6)], the transport lifetime associated with interface roughness is expressed in terms of the scattering wave vectors as

$$
\frac{1}{\tau_{\text {int }}}=\frac{\pi^{4} \hbar \Delta^{2} L^{2}}{2 m^{*} w^{6} k_{f}^{3}} \int_{0}^{2 k_{f}} \exp \left(-\frac{q^{2} L^{2}}{4}\right) \frac{q^{2} d q}{1-\sqrt{\left(q / 2 k_{f}\right)^{2}}} .
$$

The transport lifetime in this analysis is extremely sensitive to the well width $w$, so that scattering by interface roughness in wide wells is significantly lower than in narrow wells, which is due to the reduced influence the variation has on the ground-state energy $E_{0}$. This scattering mechanism potentially has a significant influence on the observed reduction in low-temperature mobility in our narrow well samples.

The total mobility can be found from the average transport lifetime in the relaxation time approximation as $^{21}$

$$
\mu=\frac{e\left\langle\tau_{\mathrm{tt}}\right\rangle}{m^{*}},
$$

where

$$
\frac{1}{\tau_{\mathrm{tr}}}=\frac{1}{\tau_{\mathrm{po}}}+\frac{1}{\tau_{\mathrm{ac}}}+\frac{1}{\tau_{\mathrm{bgi}}}+\frac{1}{\tau_{\mathrm{rii}}}+\frac{1}{\tau_{\mathrm{int}}} .
$$




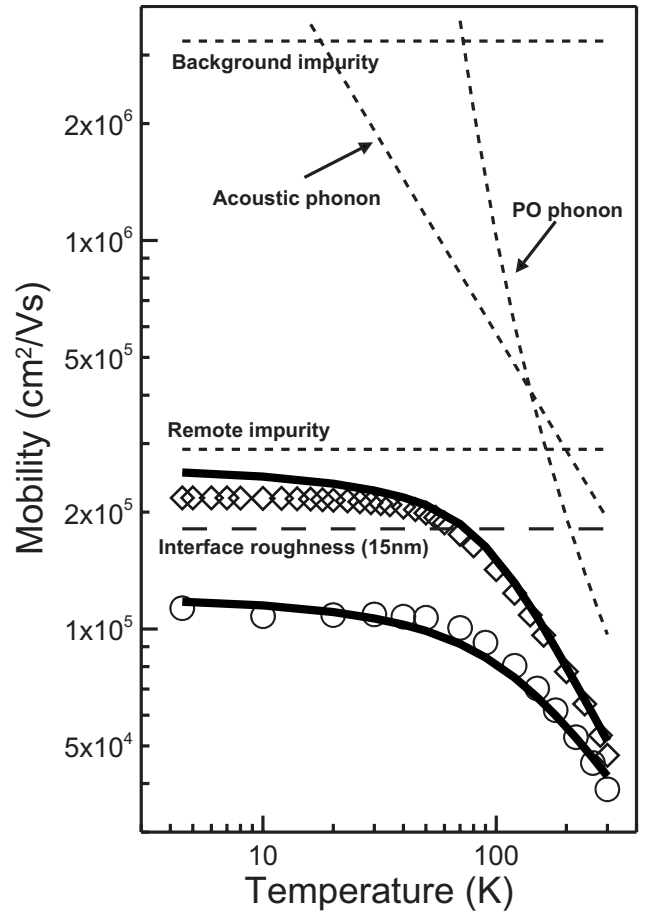

FIG. 6. Mobility for a typical $30 \mathrm{~nm}$ InSb quantum well heterostructure with a $20 \mathrm{~nm}$ spacer (open diamonds) with a theoretical fit (solid line) and a typical $15 \mathrm{~nm}$ InSb quantum well (open circles) with a theoretical fit (solid line) using the transport lifetime model. The contributions of individual scattering mechanisms are shown as dashed lines.

Figure 6 shows a comparison of this combined model [Eqs. (12) and (13)] with the temperature-dependent mobility of a typical $30 \mathrm{~nm}$ InSb quantum well heterostructure with a $20 \mathrm{~nm}$ spacer, along with the contributions of the individual scattering mechanisms outlined in Eqs. (2), (4), (7), and (8). Temperature-dependent mobility for a $15 \mathrm{~nm}$ quantum well sample is also shown, with a good fit according to the above model. The lower mobility, especially at low temperatures, can be explained by invoking interface roughness scattering [Eq. (11)]. The parameters used in the consideration of interface roughness scattering were $\Delta \approx 6.5 \AA$ (roughly two monolayers of $\mathrm{InSb}$ ) and $L \approx 20 \mathrm{~nm}$, which is consistent with observations of interface roughness in other heterostructure systems. ${ }^{23,27}$ In the narrow well sample, interface roughness scattering is the dominant mechanism at low temperatures (based on the above assumptions) and this is the only contributory mechanism shown in Fig. 6 that relates to the 15 $\mathrm{nm}$ fit. The contribution of interface roughness scattering, which uses the same parameters, is negligible for the $30 \mathrm{~nm}$ well samples due to the strong dependence of $\tau_{\text {int }}$ on the well width. The assumption of an infinite potential has been shown to introduce some error in the interface roughness scattering term for very narrow quantum wells but is more accurate in wells of width greater than $10 \mathrm{~nm} .{ }^{25}$ Nevertheless, this is an effective mechanism for explaining the difference in mobilities between our 15 and $30 \mathrm{~nm}$ well samples. The use of a Gaussian function to describe the $\Delta$ and $L$ correlations is somewhat arbitrary and is sometimes replaced with an exponentially decaying correlation function, ${ }^{26}$ for which accurate characterization of the interface is available.

The remaining parameters used in the fitting mobility model are taken from typical data for InSb (Refs. 28-31) and are $\epsilon_{s}=16.85, \epsilon_{\infty}=15.7, \Xi=30 \mathrm{eV}, \rho_{d}=5790 \mathrm{~kg} \mathrm{~m}^{-3}, v_{s}$ $=3700 \mathrm{~ms}^{-1}, \hbar \omega_{0}=25 \mathrm{meV}, m^{*}=0.0138, q_{\mathrm{TF}}=0.029 \mathrm{~nm}^{-1}$, and $^{32} n_{\text {imp }}^{3 \mathrm{D}}=5 \times 10^{15} \mathrm{~cm}^{-3}$. It is possible to achieve a good fit with the data, which suggests that the low-temperature mobility is still dominated by remote ionized impurity scattering despite the larger spacer between the well and the Te-doping plane. It is evident from Fig. 6 that the temperature dependence of mobility mainly enters through the phonon scattering, which is dominant at high temperatures. At low temperatures, the dependence becomes suppressed as the scattering becomes dominated by the impurity scattering terms and, especially in narrow wells, interface roughness scattering. In narrow-gap materials, such as InSb, the conduction band dispersion is nonparabolic, leading to a changing effective mass with energy. ${ }^{28}$ This results in temperature dependence of the effective mass, $m^{*}$, and the density of states. However, the mobility is more strongly dependent on the explicit temperature dependences in Eqs. (3) and (4), and the effects of band nonparabolicity in this context are reduced.

Figure 5 shows that the wide well samples remain somewhat dependent on $d$ and, hence, on ionized impurity scattering at $300 \mathrm{~K}$. This implies that a higher room temperature mobility is potentially achievable. This is demonstrated in Fig. 6, in which the polar optical phonon scattering limits the mobility to around $90000 \mathrm{~cm}^{2} / \mathrm{V} \mathrm{s}$. The absence of any dependence of the high-temperature mobility on the spacer for the narrow well samples (Fig. 5) may be attributed to a stronger dependence on interface roughness scattering.

\section{CONCLUSIONS}

We have examined the mobility and carrier concentration of a number of InSb-based heterostructures over a range of temperatures from 4.5 to $300 \mathrm{~K}$. Two types of structure have been considered, one with narrow $(15 \mathrm{~nm})$ quantum wells and the other with wide $(30 \mathrm{~nm})$ quantum wells. Of the two types of heterostructure, the highest low-temperature mobility is achieved by the wide well structures below $40 \mathrm{~K}$; this is found to range from 190000 to $248000 \mathrm{~cm}^{2} / \mathrm{V} \mathrm{s}$ according to the level of doping present in the barrier and the spacer size. As room temperature is approached, the mobilities converge, and at $300 \mathrm{~K}$ the wide well sample mobilities are around $50000 \mathrm{~cm}^{2} / \mathrm{V} \mathrm{s}$. The narrower well samples are found to have lower mobilities at low temperatures in the range $63000-111000 \mathrm{~cm}^{2} / \mathrm{V} \mathrm{s}$, and room temperature mobilities at low temperatures between 34500 and $39900 \mathrm{~cm}^{2} / \mathrm{V} \mathrm{s}$. This is attributed to the enhanced effect of interface roughness scattering in the narrower wells. At temperatures above $40-50 \mathrm{~K}$, the effects of phonon scattering start to play a role, with the mobility dominated by polar optical phonon scattering at temperatures approaching $300 \mathrm{~K}$. Typical carrier concentrations for these structures are in the ranges $(3.7-5.1) \times 10^{11} \mathrm{~cm}^{-2}$ at $4.5 \mathrm{~K}$ and $(5.1-8.0) \times 10^{11} \mathrm{~cm}^{-2}$ at $300 \mathrm{~K}$ for the wide well samples and $(2.8-5.7) \times 10^{11} \mathrm{~cm}^{-2}$ at $4.5 \mathrm{~K}$ and $(3.7-6.0) \times 10^{11} \mathrm{~cm}^{-2}$ at $300 \mathrm{~K}$ for the narrow well samples. 
The measured carrier concentration conforms well to predictions from Schrödinger-Poisson calculations that use appropriate parameters.

Transport lifetimes are determined for polar optical and acoustic phonon scatterings, interface roughness scattering, and scattering from charged impurities both in the 3D background and within a distributed "quasi-2D" doping layer. These are combined to give a predicted mobility, which is then compared to the measured mobility data for both types of structure. The comparison is good and is instructive in identifying mobility-limiting scattering mechanisms in the material, and the trend of the data is in good agreement with other theoretical treatments of this material heterostructure. ${ }^{30}$
The measured values of mobility are among the highest reported for room temperature III-V systems in what is a technologically important material for high-speed device applications. ${ }^{33}$ The low-temperature data are also among the highest reported for a single InSb QW material, making it an extremely interesting material system for future quantum devices. ${ }^{34}$

\section{ACKNOWLEDGMENTS}

Two of the authors (J.M.S.O. and A.M.G.) wish to acknowledge the support of the EPSRC-GB, United Kingdom.

*p.buckle@qinetiq.com

${ }^{1}$ T. Ashley, A. B. Dean, C. T. Elliott, G. J. Pryce, A. D. Johnson, and H. Willis, Appl. Phys. Lett. 66, 481 (1995).

${ }^{2}$ A. M. Song, A. Lorke, A. Kriele, J. P. Kotthaus, W. Wegscheider, and M. Bichler, Phys. Rev. Lett. 80, 3831 (1998).

${ }^{3}$ S. A. Solin, D. R. Hines, A. C. H. Rowe, J. S. Tsai, Y. A. Pashkin, S. J. Chung, N. Goel, and M. B. Santos, Appl. Phys. Lett. 80, 4012 (2002).

${ }^{4}$ V. P. Kunets, W. T. Black, Y. I. Mazur, D. Guzun, G. J. Salamo, N. Goel, D. Mishima, D. A. Deen, S. Q. Murphy, and M. B. Santos, J. Appl. Phys. 98, 014506 (2005).

${ }^{5}$ Y. Hirayama, T. Saku, S. Tarucha, and Y. Horikoshi, Appl. Phys. Lett. 58, 2672 (1991).

${ }^{6}$ F. H. L. Koppens, C. Buizert, K. J. Tielrooij, I. T. Vink, K. C. Nowack, T. Meunier, L. P. Kouwenhoven, and L. M. K. Vandersypen, Nature (London) 442, 766 (2006).

${ }^{7}$ M. T. Bjork, A. Fuhrer, A. E. Hansen, M. W. Larsson, L. E. Froberg, and L. Samuelson, Phys. Rev. B 72, 201307(R) (2005).

${ }^{8}$ K. J. Goldammer, S. J. Chung, W. K. Liu, M. B. Santos, J. L. Hicks, S. Raymond, and S. Q. Murphy, J. Cryst. Growth 201/ 202, 753 (1999).

${ }^{9}$ N. Goel, J. Graham, J. C. Keay, K. Suzuki, S. Miyashita, M. B. Santos, and Y. Hirayama, Physica E (Amsterdam) 26, 455 (2005).

${ }^{10}$ J. M. S. Orr, P. D. Buckle, M. Fearn, C. J. Bartlett, L. Buckle, and T. Ashley, AIP Conf. Proc. 893, 729 (2007).

${ }^{11}$ J. M. S. Orr, P. D. Buckle, M. Fearn, P. J. Wilding, C. J. Bartlett, M. T. Emeny, L. Buckle, and T. Ashley, Semicond. Sci. Technol. 21, 1408 (2006)

${ }^{12}$ A. M. Gilbertson, J. M. S. Orr, P. D. Buckle, S. Clowes, M. Fearn, C. J. Storey, L. Buckle, L. F. Cohen, and T. Ashley, Phys. Rev. B 76, 085306 (2007).

${ }^{13}$ A. D. Andreev (unpublished).

${ }^{14}$ J. Singh, Physics of Semiconductors and Their Heterostructures (McGraw-Hill, New York, 1993).

${ }^{15}$ F. A. Riddoch and B. K. Ridley, J. Phys. C 16, 6971 (1983).

${ }^{16}$ V. K. Arora and A. Naeem, Phys. Rev. B 31, 3887 (1985).

${ }^{17}$ B. K. Ridley, J. Phys. C 15, 5899 (1982).

${ }^{18}$ W. Walukiewicz, H. E. Ruda, J. Lagowski, and H. C. Gatos, Phys. Rev. B 30, 4571 (1984).

${ }^{19}$ J. Bardeen and W. Shockley, Phys. Rev. 80, 72 (1950).

${ }^{20}$ B. J. F. Lin, D. C. Tsui, M. A. Paalanen, and A. C. Gossard, Appl. Phys. Lett. 45, 695 (1984).

${ }^{21}$ P. J. Price, Ann. Phys. (N.Y.) 133, 217 (1981).

${ }^{22}$ J. H. Davies, The Physics of Low-Dimensional Semiconductors: An Introduction (Cambridge University Press, Cambridge, 1997).

${ }^{23}$ W. P. Hong, J. Singh, and P. K. Bhattacharya, IEEE Electron Device Lett. 7, 480 (1986).

${ }^{24}$ H. Sakaki, T. Noda, K. Hirakawa, M. Tanaka, and T. Matsusue, Appl. Phys. Lett. 51, 1934 (1987).

${ }^{25}$ U. Penner, H. Rücker, and I. N. Yassievich, Semicond. Sci. Technol. 13, 709 (1998).

${ }^{26}$ S. M. Goodnick, D. K. Ferry, C. W. Wilmsen, Z. Liliental, D. Fathy, and O. L. Krivanek, Phys. Rev. B 32, 8171 (1985).

${ }^{27}$ C. R. Bolognesi, H. Kroemer, and J. H. English, Appl. Phys. Lett. 61, 213 (1992).

${ }^{28}$ Y. Shao, S. A. Solin, and L. R. Ram-Mohan, APS Meetings, 2006, p. 35008.

${ }^{29}$ S. M. Sze, Physics of Semiconductor Devices, 2nd ed. (Wiley, New York, 1981).

${ }^{30}$ Y. Shao, S. A. Solin, L. R. Ram-Mohan, and Keon-Ho Yoo, J. Appl. Phys. 101, 123704 (2007).

${ }^{31}$ G. Harbeke, O. Madelung, and U. Rössler, Functional Relationships in Science and Technology, New Series, Landolt-Börnstein, New Series, Group III, Vol. 17, Pt. A (Springer-Verlag, Berlin, 1982).

${ }^{32}$ G. J. Nott, P. C. Findlay, J. G. Crowder, C. R. Pidgeon, C. T. Elliott, T. Ashley, A. D. Johnson, J. P. R. Wells, I. V. Bradley, B. N. Murdin, and A. M. White, J. Phys.: Condens. Matter 12, L731 (2000); the background impurity density parameter is modified here to account for the higher aluminum fraction in the present work.

${ }^{33}$ T. Ashley, A. R. Barnes, L. Buckle, S. Datta, A. B. Dean, M. T. Emeny, M. Fearn, D. G. Hayes, K. P. Hilton, R. Jefferies, T. Martin, K. J. Nash, T. J. Phillips, W. A. Tang, P. J. Wilding, and R. Chau, Proceedings of the Seventh International Conference on Solid-State and Integrated Circuits Technology (2004), Vol. 3, p. 2253.

${ }^{34}$ J. M. S. Orr, P. D. Buckle, M. Fearn, C. J. Storey, L. Buckle, and T. Ashley, New J. Phys. 9, 261 (2007). 